\title{
Coréférence événementielle entre deux phrases ${ }^{1}$
}

\author{
Laurence Danlos
}

LORIA / LATTICE

\section{Résumé}

Les travaux sur les relations temporelles entre deux éventualités $e_{1}$ et $e_{2}$ supposent toujours que $e_{1} \neq e_{2}$. Nous nous concentrerons sur les cas où $e_{1}=e_{2}$, c'est-à-dire sur la coréférence événementielle. À la différence de la coréférence entre objets, la coréférence événementielle n'a pas été étudiée en détail, excepté pour un groupe (pro)nominal référant à un événement. Nous étudierons ici la coréférence événementielle entre deux phrases. Cette étude mettra en avant des phénomènes linguistiques inhabituels (e. g. coréférence entre des éléments existentiellement quantifiés). Ces phénomènes doivent être pris en compte en compréhension et en génération de texte. Ils nous conduiront à introduire et définir de nouvelles relations de discours qui seront discutées dans le cadre de la SDRT.

\section{Introduction}

Les travaux sur les relations temporelles entre deux éventualités $e_{1}$ et $e_{2}$ examinent exclusivement si une des éventualités précède, inclut ou chevauche l'autre [Moens et Steedman, 1988, Asher, 1993, Pustejovsky, 1995]. Toutes ces relations temporelles supposent que $e_{1} \neq e_{2}$. Nous nous concentrerons sur les cas où $e_{1}=e_{2}$, c'est-à-dire sur la coréférence événementielle.

A l'inverse de la coréférence entre objets, la coréférence entre événements n'a guère été étudiée en détail, excepté pour un groupe (pro)nominal référant à un événement, voir parmi d'autres [Webber, 1988, Asher, 1993]. Néanmoins, il existe bien d'autres cas de coréférence événementielle puisque la description d'un événement $e$ peut être de trois types : c'est soit un groupe (pro)nominal, soit une phrase, soit une abstraction qui n'est pas linguistiquement réalisée. Ce dernier cas apparaît quand $e$ est un sous-événement d'un événement complexe. Le nombre de cas potentiels de coréférence événementielle entre deux descriptions successives $D_{1}$ et $D_{2}$ du même événement $e$ est donc $3^{2}=9$. Cet article se concentre sur la coréférence événementielle pour les cas où $\mathrm{D}_{1}$ et $\mathrm{D}_{2}$ sont toutes deux des phrases (notées $P_{i}$ ) comme en (1) et $(2)^{2}$. Nous

\footnotetext{
${ }^{1}$ Je voudrais remercier Bertrand Gaiffe qui m'a aidée dans la formalisation des données en SDRT et Michel Cosse pour ses commentaires fructueux.

${ }^{2}$ Dans [Danlos, 2000], nous avons étudié la coréférence événementielle quand $\mathrm{D}_{i}$ est une phrase et $\mathrm{D}_{j}(\mathrm{i} \neq \mathrm{j})$ n'est pas réalisée syntaxiquement. Plus précisément, le cas où $\mathrm{D}_{j}$ est la description abstraite du sous-événement causal de l'événement complexe décrit par un verbe causatif et $P i$ la phrase décrivant la cause est examinée, voir (i) et (ii).

(i) Fred a fêlé la carafe. Il l'a heurtée contre l'évier.

(ii) Fred a heurté la carafe contre l'évier. Il l'a fêlée.
} 
supposerons qu'une phrase $P_{i}$ réfère à un événement unique $e_{i}{ }^{3}$. Ceci signifie que nous étudierons les discours $P_{1}$. $P_{2}$. tels que $P_{1}$ et $P_{2}$ réfèrent au même événement unique $e$.

Fred a soigné un arbre. Il a élagué un cèdre.

Fred a élagué un cèdre. Il a donc soigné un arbre.

L'étude de la coréférence événementielle demande la définition de deux types de relation de coréférence selon la quantité d'information transmise :

Deux descriptions successives $D_{1}$ et $D_{2}$ de la même entité x (événement ou objet) sont dans une relation de particularisation notée $D_{2}=P A R T\left(D_{1}\right)$ ssi $D_{2}$ apporte au moins une information nouvelle sur x par rapport à l'information connue de $D_{l}$.

Deux descriptions successives $D_{1}$ et $D_{2}$ de la même entité x (événement ou objet) sont dans une relation de généralisation notée $D_{2}=G E N\left(D_{1}\right)$ ssi $D_{2}$ n'apporte aucune nouvelle information sur $x$.

Illustrons ces deux types de coréférence sur le cas bien connu où $\mathrm{x}$ est un objet. $\mathrm{D}_{1}$ et $\mathrm{D}_{2}$ sont alors des groupes (pro)nominaux. Dans (3a), le GN démonstratif cette New-Yorkaise = PART(une actrice). Dans (3b), le pronom elle = GEN(une actrice) et dans (3c) le GN défini ou démonstratif la / cette artiste $=\mathrm{GEN}($ une actrice $)$ puisque artiste est un hyperonyme de actrice .

(3)a Fred a pris un verre avec une actrice. Cette New-Yorkaise irrite Marie.

b $\quad$ Fred a pris un verre avec une actrice. Elle irrite Marie.

c $\quad$ Fred a pris un verre avec une actrice. La / Cette artiste irrite Marie.

Dans les sections suivantes, nous montrerons que la relation de coréférence événementielle est du type particularisation dans (1), du type généralisation dans (2). Nous présenterons une étude linguistique de ces discours et formaliserons les données dans le cadre de la SDRT [Asher, 1993].

Notation : Le symbole $X_{i}^{r}$ avec $\mathrm{i}=1$ ou 2 dénote le prédicat ou un argument ou un modifieur dans $P_{i}$ avec le rôle $\mathrm{r}$; pour un argument $\mathrm{r}=$ agent, patient $\ldots$; pour un modifieur $\mathrm{r}=$ temps, lieu, ... ; pour le prédicat, $r$ est non pertinent.

\section{Discours particularisants et généralisants}

\subsection{Discours particularisants}

Une relation de coréférence événementielle de type particularisation s'observe dans "des discours particularisants" (dorénavant DP) comme (1) ou (4).

Fred a sali un vêtement. Il a taché une chemise.

Dans (4), le même événement est décrit dans les deux phrases, avec plus d'information dans $P_{2}$. $P_{2}$ particularise $P_{1}$ parce que son prédicat est un hyponyme du prédicat de $P_{1}$ ( tacher < salir) et que son objet (indéfini) est un hyponyme de l'objet (indéfini) de $P_{l}$ (chemise $<$ vêtement) (le sujet

\footnotetext{
${ }^{3}$ Ceci est une simplification ; en effet une phrase avec un argument pluriel peut référer à plusieurs événements. Par exemple, la première phrase dans (i) ci-dessous avec l'argument pluriel deux vêtements réfèrent en fait à deux événements qui sont décrits dans la deuxième et troisième phrase. Dans cet article, les arguments pluriels seront laissés de côté.
}

(i) Fred a sali deux vêtements. Il a taché une chemise lundi. Il a déchiré une cravate mardi. 
de $P_{2}$ est une anaphore (pronominale) du sujet de $\left.P_{1}\right)^{4}$.

Le discours (4) (quand il est compris comme un discours complet) peut seulement être interprété comme un DP avec une relation de coréférence événementielle de type particularisation. En effet, si deux événements étaient impliqués, cela serait explicitement indiqué soit par un connecteur soit par deux adjoints différents : dans (5a), ensuite implique $e_{1}<$ $e_{2}$; dans (5b), aussi implique $e_{1} \neq e_{2}$; dans (5c), les deux adjoints hier et aujourd'hui impliquent $e_{1}<e_{2}$. Nous dirons que les discours comme (5) incluent "un marqueur de non coréférentialité".

b $\quad$ Fred a sali un vêtement. Il a aussi taché une chemise.

Fred a sali un vêtement. Ensuite, il a taché une chemise.

c $\quad$ Fred a sali un vêtement hier. Il a taché une chemise aujourd'hui.

Néanmoins, l'interprétation de particularisation de (4) peut être explicitement marquée, comme en (6) avec le connecteur plus précisément (qui peut être appelé "un marqueur de coréférentialité").

Fred a sali un vêtement. Plus précisément, il a taché une chemise.

Notons cependant qu'une troisième phrase prolongeant (4) peut changer l'interprétation des deux premières phrases. Dans (7), avec aussi dans $P_{3}$, aussi est sous-entendu dans $P_{2}$, donc $P_{1}$ et $P_{2}$ ne réfèrent pas au même événement. Dans le reste de cet article, seuls les discours de deux phrases considérés comme des discours complets seront étudiés.

Fred a sali un vêtement. Il a taché une chemise. Il a aussi déchiré une cravate.

Le DP dans (4) donne lieu à un phénomène inhabituel : les deux GN indéfinis un vêtement et une chemise sont coréférents. Ceci est inhabituel puisqu'il est généralement admis [Kamp et Reyle, 1993, Corblin, 1995] qu'un GN indéfini a une lecture existentielle (c'est-à-dire introduit un nouveau référent de discours) et donc que deux GN indéfinis ne sont pas coréférents ${ }^{5}$. C'est le cas en (8) dans lequel deux vêtements sont en jeu, tandis qu'un seul vêtement est en jeu en (4). Nous verrons dans la Section 6 comment calculer la coréférence de deux GN indéfinis dans les DP.

Fred a sali un vêtement. Joe a taché une chemise.

Un discours comme (4) dans lequel au moins un élément de $P_{2}$ spécifie un élément correspondant dans $P_{1}$ est appelé "un DP par spécification". On observe un autre type de DP dans (9) où le modifieur pendant le dîner de $P_{2}$ fournit une information temporelle sur $e$ (les autres éléments $\mathrm{X}_{2}{ }^{r}$ de $P_{2}$ anaphorisent ou répètent les éléments correspondants $\mathrm{X}_{1}{ }^{r}$ de $P_{1}$ ).

Fred a taché une chemise. Il l'a tachée pendant le dîner.

Un discours comme (9) dans lequel au moins un élément $\mathrm{X}_{2}{ }^{r}$ de $P_{2}$ n'a aucun élément correspondant $\mathrm{X}_{1}{ }^{r}$ dans $P_{1}$ est appelé "un DP par adjonction". Spécification et adjonction peuvent être combinées : dans le DP (10), chemise spécifie vêtement et l'adjoint de temps dans $P_{2}$ n'a aucun élément correspondant dans $P_{l}$.

Fred a taché un vêtement. Il a taché une chemise pendant le dîner.

À première vue, on obtient donc un DP basé sur des connaissance lexicales quand chaque

\footnotetext{
${ }^{4}$ Les deux phrases de (4) sont au même temps, ce qui est exigé pour la coréférence événementielle.

${ }^{5}$ Il existe quelques cas où deux GN indéfinis sont coréférents, voir (i) et (ii) emprunté à [Corblin, 1994].

(i) Un homme est arrivé. C'était un New-Yorkais.

(ii) Un homme, un New-Yorkais, est arrivé.
}

Cependant, les exemples présentés ici avec deux GN indéfinis coréférents n'ont pas été décrits dans la littérature à notre connaissance. 
élément $\mathrm{X}_{2}{ }^{r}$ de $P_{2}$ est un hyponyme, une anaphore ou une répétition d'un élément correspondant $\mathrm{X}_{1}{ }^{r}$ de $P_{1}$ ou bien n'a aucun élément correspondant $\mathrm{X}_{1}{ }^{r}$ dans $P_{1}$.

Des exemples plus naturels de DP sont observés quand entrent en jeu des connaissances extralinguistiques, (11). Ces DP sont basés sur une notion d'hyperonymie étendue, à savoir $X$ complimenter $Y>X$ dire à $Y q u ' Y$ être joli dans (11a) et $X$ être bilingue $>X$ parler deux langues dans (11b). Ces relations d'hyperonymie étendue reposent sur des connaissances culturelles ou encyclopédiques. Cependant, comme nous ne faisons pas une étude de sémantique lexicale, nous n'utiliserons que des exemples artificiels de DP basés sur des connaissances purement lexicales, évitant ainsi le bruit qui serait introduit par des discussions sur la notion d'hyperonymie étendue.

(11)a Fred a complimenté Sue. Il lui a dit qu'elle est jolie.

b Fred est bilingue. Il parle français et chinois.

Notre étude linguistique n'est donc pas basée sur un corpus. Néanmoins il est facile de trouver des exemples réels de DP, comme (12) (Le Monde du 15/12/2000).

Al Gore a, pendant toute cette période, fait montre d'une indéniable ténacité. Jamais, il n'a baissé les bras après un coup du sort ....

Pour récapituler, en compréhension de texte, il doit être calculé qu'il y a un seul vêtement et un seul événement décrit dans $(4)^{6}$. En génération de texte, il doit être déterminé dans quelles conditions un discours comme (4) peut être énoncé et quelles formes linguistiques doivent être produites [Roussarie, 2000].

\subsection{Discours généralisants}

Une relation de coréférence événementielle de type généralisation s'observe dans "des discours généralisants" (dorénavant DG) comme (13).

(13)a Fred a taché une chemise. Il a donc sali un vêtement.

b Fred a assassiné Sue. Il a donc commis un crime.

Alors qu'un DP se situe principalement au niveau informationnel, un DG se situe principalement au niveau intentionnel : la première phrase dans (13a) ou (13b) décrit ce que le locuteur veut que l'interlocuteur sache d'un événement $e$, la deuxième étant une reformulation de $e$. Par définition, une reformulation ne peut pas apporter d'information nouvelle, mais elle peut présenter un événement sous un jour nouveau, par exemple, si le locuteur a l'intention de forger des liens avec d'autres données, (14).

Fred a assassiné Sue. Il a donc commis un crime pour lequel il sera jugé devant les

(13a) est obtenu à partir de (4) en inversant l'ordre des phrases (et les relations anaphoriques) et en introduisant un connecteur comme donc avec une valeur épistémique [Rossari et Jayez, 1996]. $P_{2}$ généralise $P_{1}$ parce que salir et vêtement sont respectivement des hyperonymes des éléments correspondants de $P_{1}$. Dans (13b), $P_{2}$ généralise $P_{1}$ parce que le prédicat complexe commettre un crime est un hyperonyme de assassiner.

À première vue, on obtient donc un DG basé sur des connaissance lexicales quand chaque élément $\mathrm{X}_{2}{ }^{r}$ de $P_{2}$ est un hyperonyme, une anaphore ou une répétition d'un élément correspondant $\mathrm{X}_{1}{ }^{r}$ de $P_{1}$.

Comme pour les DP, des exemples plus naturels de DG sont observés quand la notion

\footnotetext{
${ }^{6}$ Voir aussi [Humphreys et alii] pour la coréférence événementielle dans un système d'extraction d'informations.
} 
d'hyperonymie étendue est en jeu, (15). Cependant, nous n'utiliserons que des DG basés sur des connaissances purement lexicales.

(15)a Fred a dit à Marie qu'elle est jolie. Il l'a donc complimentée.

b Nous avons la Chambre, nous avons le Sénat et nous avons la Maison Blanche. Ce qui veut dire que nous contrôlons l'ordre du jour. (Le Monde du 15/12/2000)

\subsection{Discours $P_{1} . P_{2}$ avec une coréférence événementielle}

Dans le reste de cet article, tout discours qui n'implique pas de relation de coréférence événementielle sera marqué avec le signe \#, qu'il soit incohérent (ininterprétable) ou qu'il ait une interprétation sans coréférence événementielle.

Dans les DP et DG, chaque élément $\mathrm{X}_{2}{ }^{r}$ de $P_{2}$ qui a un élément correspondant $\mathrm{X}_{1}{ }^{r}$ de $P_{1}$ est dans une relation lexico-sémantique (hyponymie, hyperonymie, anaphore, identité ou synonymie $^{7}$ ) avec cet élément. Ce sont les deux seuls types de discours $P_{1}$. $P_{2}$. dans lesquels les deux phrases réfèrent au même événement.

- En premier lieu, si un (ou plusieurs) élément(s) de $P_{2}$ avec un élément correspondant dans $P_{1}$ n'est pas dans une relation lexico-sémantique avec cet élément, les deux phrases ne peuvent pas référer au même événement : c'est le cas pour (16a) avec les deux prédicats tacher et déchirer, pour (16b) avec les deux objets une chemise et une cravate et pour (16c) avec les deux adjoints de temps hier et aujourd'hui.

(16)a \# Fred a taché une chemise. Il l'a déchirée.

b \# Fred a taché une chemise. Il a taché une cravate.

c \# Fred a embrassé Sue hier. Il l'a embrassée aujourd'hui.

- Deuxièmement, si chaque élément de $P_{2}$ est dans une relation lexico-sémantique avec un élément correspondant de $P_{l}$, mais si un élément est dans la relation hyponymique tandis qu'un autre est dans la relation hyperonymique, les deux phrases ne peuvent pas référer au même événement. Dans (17a) ou (17b), dans lesquels le prédicat de $P_{2}$ est un hyponyme du prédicat de $P_{1}$, tacher < salir, tandis que l'objet (indéfini) de $P_{2}$ est un hyperonyme de l'objet (indéfini) de $P_{l}$, vêtement > chemise, aucune coréférence événementielle n'est en jeu. Cependant, nous verrons dans la Section 5.2 que certains DP incluent et des hyperonymes et des hyponymes dans $P_{2}$.

(17)a \# Fred a sali une chemise. Il a taché un vêtement.

b \# \# Fred a sali une chemise. Il a donc taché un vêtement.

- Finalement, les deux phrases ne doivent pas apporter la même quantité d'information ou le même point de vue sur l'information transmise. La répétition pure et simple dans $P_{2}$ de l'information transmise dans $P_{1}$ donne lieu à un discours incohérent, (18a) ou (18b). Deux directions sont donc disponibles : ou bien apporter de l'information nouvelle, ce qui est le rôle des DP, ou bien présenter l'information en des termes plus généraux afin de forger des liens avec d'autres données, ce qui est le rôle des DG.

\footnotetext{
${ }^{7}$ Les exemples de DP et de DG qui impliquent une relation de synonymie ne seront pas illustrés dans cet article. Le lecteur vérifiera que nos conclusions restent valables pour ces exemples, même avec les paires converses de synonymes (recevoir et donner), (i) et (ii).

(i) Marie a reçu un cadeau de Fred. Il lui a donné un collier.

(ii) Fred a donné un collier à Marie. Elle a donc reçu un cadeau de lui.
} 
(18)a \# Fred a taché une chemise. Il l'a tachée.

b \# Fred a taché une chemise. Il l'a donc tachée.

\subsection{Hypothèse}

A partir de ces observations, l'hypothèse suivante peut être avancée :

Une relation de coréférence événementielle s'observe dans un discours $P_{1} . P_{2}$. seulement si :

- Chacun chaque élément dans $P_{2}$ est un hyponyme, une anaphore ou la répétition d'un élément correspondant dans $P_{1}$ (s'il existe) (une relation de particularisation est alors en jeu),

- Ou si chaque élément dans $P_{2}$ (comportant un connecteur comme donc) est un hyperonyme, une anaphore ou la répétition d'un élément correspondant dans $P_{1}$ (une relation de généralisation est alors en jeu).

On peut traduire cette hypothèse en termes "d'implication" :

- Dans un DP, $P_{2}$ "implique" $P_{1}$ : d'une façon informelle, tacher une chemise implique salir un vêtement, donc Fred a taché une chemise implique Fred a sali un vêtement dans (4).

- Dans un DG, $P_{1}$ "implique" $P_{2}$ : d'une façon informelle, dans (13a) Fred a taché une chemise implique Fred a sali un vêtement.

Dans la section suivante, nous allons présenter, dans le cadre de la DRT [Kamp et Reyle, 1993], une définition formelle de cette notion "d'implication" entre deux DRS. Dans la Section 4, nous montrerons que les conditions pour observer un DG composé de deux phrases sont basées sur une relation d'implication entre les DRS associés aux deux phrases. Par contre, nous verrons dans la Section 5 que la question est plus complexe pour les DP.

\section{Implication entre deux DRS}

En termes logiques, une relation d'hyponymie entre noms comme cèdre < arbre se traduit en :

$$
\forall x \operatorname{cèdre}(x) \Rightarrow \operatorname{arbre}(x)
$$

Donc, l'implication suivante est valable :

$$
\exists x \operatorname{cèdre}(x) \quad \Rightarrow \quad \exists y \operatorname{arbre}(y)
$$

De la même façon, une relation d'hyponymie entre verbes comme élaguer < soigner donne :

$$
\forall u, v \exists e_{1} \quad \text { élaguer }\left(e_{1}, u, v\right) \Rightarrow \exists e_{2} \quad \operatorname{soigner}\left(e_{2}, u, v\right)
$$

Et une relation d'hyponymie (étendue) entre adverbiaux comme ce matin < aujourd'hui (ce matin = aujourd'hui dans la matinée) donne :

$$
\forall e_{1} c e-\operatorname{matin}\left(e_{1}\right) \Rightarrow \text { aujourd' } \operatorname{hui}\left(e_{1}\right)
$$

Nous employons aussi l'implication suivante qui est toujours vraie :

$\forall x P(x) \wedge Q(x) \Rightarrow P(x)$ 
Par exemple :

$$
\exists e_{1} \quad \text { élaguer }\left(e_{1}, f, x\right) \wedge \text { amoureusement }\left(e_{1}\right) \Rightarrow \exists e_{1} \text { élaguer }\left(e_{1}, f, x\right)
$$

Finalement, pour traiter les anaphores, nous employons des schémas d'implication comme :

$$
\exists x \operatorname{Fred}(x) \Rightarrow \exists y \operatorname{humain}(y) \wedge \operatorname{mâle}(y)
$$

A partir de ces implications logiques, nous pouvons définir une relation d'implication entre deux DRS, notée $\rightrightarrows$, quand les prédicats lexicaux dans les conditions des DRS sont dans des relations d'hyponymie. Sans entrer dans les détails formels, cette relation d'implication est illustrée dans l'exemple suivant dans lequel $k_{1}$ correspond à Fred a amoureusement élagué un cèdre ce matin et $k_{1}^{\prime}$ à Il a soigné un arbre aujourd'hui.

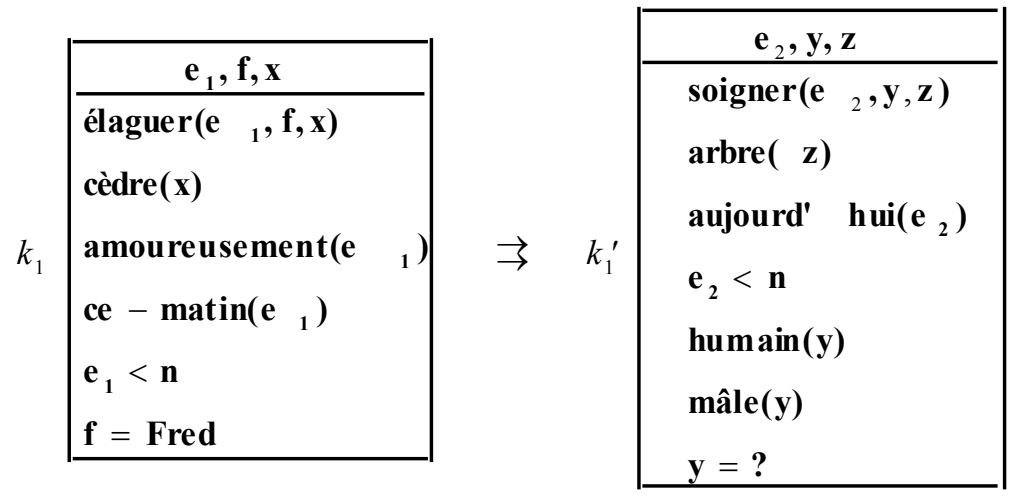

Notons que les conditions comme $y=$ ? dans $k_{1}^{\prime}$ sont ignorées. Evidemment, deux DRS qui diffèrent seulement par les noms de leurs variables liées sont considérés comme identiques (cette relation d'identité est notée $\cong$ ).

\section{Conditions pour observer un DG}

Nous allons montrer que les conditions pour observer un DG composé de deux phrases sont les suivantes (cet ensemble de conditions est noté $C_{G D}$ ) :

Un discours composé de deux phrases est un $D G$ ssi (i) $P_{2}$ inclut éventuellement un connecteur épistémique comme donc ${ }^{8}$ et (ii) les DRS $k_{1}$ et $k_{2}$ associées respectivement à $P_{1}$ et $P_{2}$ sont telles que $k_{1} \rightrightarrows k_{1}^{\prime}$ avec $k_{1}^{\prime} \cong k_{2}\left(\right.$ et $\left.\neg k_{1} \cong k_{2}\right)$.

$C_{G D}$ est évidemment respecté dans un exemple de base de DG, (2) répété en (19).

Fred a élagué un cèdre. Il a donc soigné un arbre.

La condition $\neg k_{1} \cong k_{2}$ est nécessaire pour exclure des exemples tels que (18b) ou (20), dans lesquels $P_{2}$ répète l'information transmise dans $P_{1}$. Pour de tels cas, $k_{1} \cong k_{2}$.

\# Fred a élagué un cèdre. Il l'a donc élagué.

Dans des exemples comme (17b) et (21), qui ne sont pas des DG, $C_{G D}$ n'est pas respecté parce que le verbe de $P_{2}$ est un hyponyme du verbe de $P_{1}$ (élaguer < soigner).

\footnotetext{
${ }^{8}$ La présence de donc n'est pas obligatoire : (i), emprunté à [Corblin, 1995], présente une relation de coréférence événementielle de type généralisation.
}

(i) Fred a consulté le docteur Larsen. Il a consulté un docteur parce qu'il se sentait fatigué. 
Considérons maintenant l'adjonction de modifieurs. Dans (19), il est possible d'insérer n'importe quel modifieur dans $P_{1}$ tout en maintenant l'interprétation de généralisation. (22a) avec hier dans $P_{1}$ est un DG. $k_{1}$, la DRS pour la première phrase de (22a), inclut la condition hier $\left(e_{1}\right)$ qui n'a pas d'équivalent dans $k_{2}$. Donc, $C_{G D}$ est respecté dans $(22 \mathrm{a})^{9}$. De la même façon, (22b) avec un modifieur évaluatif dans $P_{1}$ (amoureusement) et aucun modifieur dans $P_{2}$ est aussi un DG pour lequel $C_{G D}$ est respecté.

(22)a Fred a élagué un cèdre hier. Il a donc soigné un arbre.

b $\quad$ Fred a amoureusement élagué un cèdre. Il a donc soigné un arbre.

Il n'est possible d'insérer un adjoint dans $P_{2}$ que s'il généralise un adjoint dans $P_{1}$. (23a) avec ce matin dans $P_{1}$ et aujourd'hui dans $P_{2}$ est un DG. Comme ce matin peut être vu comme un hyponyme de aujourd'hui, $C_{G D}$ est respecté dans (23a). Par contre, (23b) avec hier dans $P_{2}$ et aucun adjoint de temps dans $P_{l}$ n'est pas un DG et $C_{G D}$ n'est pas respecté dans (23b).

(23)a Fred a élagué un cèdre ce matin. Il a donc soigné un arbre aujourd'hui.

b \# Fred a élagué un cèdre. Il a donc soigné un arbre hier.

Le lecteur vérifiera que les DG basés sur des relations d'hyponymie étendue, (15), sont tels que $C_{G D}$ est respecté.

\section{Conditions pour observer un DP}

Nous allons d'abord examiner les DP avec un DG correspondant, c'est-à-dire les DP tels qu'un DG peut être créé en inversant l'ordre des phrases et les relations anaphoriques (et en introduisant éventuellement un connecteur comme donc). Les conditions pour observer de tels DP, à savoir $C_{P D}$, sont symétriques de $C_{G D}$ et sont donc basées sur la notion d'implications entre les DRS représentant les deux phrases. Ensuite, nous examinerons les DP sans DG correspondant, pour lesquels il n'y a pas d'implication entre les DRS des deux phrases.

\subsection{DP avec un DG correspondant}

Les conditions pour observer un DP avec un DG correspondant sont les suivantes (cet ensemble de conditions est noté $C_{P D}$ ) :

Un discours composé de deux phrases est un DP ssi (i) $P_{2}$ n'inclut aucun marqueur de non coréférentialité (voir Section 2.1) mais inclut éventuellement un marqueur de coréférentialité et (ii) les DRS $k_{1}$ et $k_{2}$ associées respectivement à $P_{1}$ et $P_{2}$ sont telles que $k_{2} \rightrightarrows k_{2}^{\prime}$ avec $k_{2}^{\prime} \cong k_{1}$ (et $\neg k_{1} \cong k_{2}$ ).

Les exemples de (24) sont tous des DP avec un DG correspondant, respectivement (19), (22a), (22b) et (23a). $C_{P D}$ est respecté pour ces discours comme $C_{G D}$ est respecté pour le DG correspondant.

(24)a Fred a soigné un arbre. Il a élagué un cèdre.

b Fred a soigné un arbre. Il a élagué un cèdre hier.

c Fred a soigné un arbre. Il a amoureusement élagué un cèdre.

d Fred a soigné un arbre aujourd'hui. Il a élagué un cèdre ce matin.

\footnotetext{
${ }^{9}$ Dans $k_{2}$, La condition $e_{2}<n$ pourrait être réécrite $P A S S E ́\left(e_{2}\right)$. Il suffit alors de considérer hier comme un hyponyme de $P A S S E ́$ pour voir que $C_{G D}$ est respecté dans (22a).
} 


\subsection{DP sans DG correspondant}

Le premier type d'exemples de DP sans DG correspondant est illustré dans (25a) : (25a) est un DP (les deux phrases réfèrent au même événement avec plus d'information dans $P_{2}$ ), tandis que le discours obtenu en inversant l'ordre des phrases et les relations anaphoriques, à savoir (23b) répété dans (25b), n'est pas un DG. $C_{P D}$ n'est pas respecté dans (25a) de la même manière que $C_{G D}$ n'est pas respecté dans $(25 \mathrm{~b})$.

(25)a Fred a soigné un arbre hier. Il a élagué un cèdre.

b \# Fred a élagué un cèdre. Il a donc soigné un arbre hier.

$P_{1}$ dans (25a) inclut un adjoint de temps (hier) qui n'est pas répété dans $P_{2}$, bien qu'il soit compris que l'événement d'élagage a eu lieu hier et que c'est le même événement que celui de soigner. Cela signifie que la deuxième phrase d'un DP doit apporter au moins une information nouvelle, mais ne doit pas nécessairement répéter toute l'information transmise dans la première phrase. Par contre, la deuxième phrase d'un DG ne peut apporter aucune information nouvelle.

Le deuxième type d'exemples de DP sans DG correspondant est illustré dans (26a) : (26a) est un DP bien que le verbe de $P_{2}$ soit un hyperonyme du verbe de $P_{1}$. Il n'a aucun DG correspondant - (26b) n'est pas un DG - et $C_{P D}$ n'est pas respecté. (26a) est paraphrasé par (26c) avec la forme anaphorique faire cela.

(26)a Fred a élagué un cèdre. Il l'a soigné (s'en est occupé) aujourd'hui.

b \# Fred a soigné (s'est occupé d') un cèdre aujourd'hui. Il l'a donc élagué.

c Fred a élagué un cèdre. Il a fait cela aujourd'hui.

Dans (26a) ou (26c), la nouvelle information dans $P_{2}$ vient de l'adverbe aujourd'hui qui ne peut pas être omis : les discours (27) sans un tel adverbe sont inacceptables.

(27)a \# Fred a élagué un cèdre. Il l'a soigné.

b \# Fred a élagué un cèdre. Il l'a fait.

Dans (26a), les arguments de $P_{2}$ sont des anaphores des arguments correspondants de $P_{1}$. Considérons les exemples (28) qui sont identiques à (26a) sauf qu'un argument (le patient) n'est pas une anaphore du patient dans $P_{1}: \operatorname{Arg}_{2}{ }^{\text {Patient }}$ est respectivement une répétition, un hyperonyme et un hyponyme d'Arg ${ }_{1}^{\text {Patient }}$ dans (28a), (28b) et (28c). Ces exemples ne sont pas clairement interprétés avec une relation de coréférence événementielle, bien que cette interprétation ne soit pas exclue (avec une prosodie spécifique, par exemple accent sur aujourd'hui dans (28a) et (28b), sur cèdre dans (28c)), d'où le signe ? les précédant. L'interprétation coréférentielle est plus facilement accessible avec le marqueur de coréférentialité plus précisément, (28d).

(28)a ? Fred a élagué un cèdre. Il a soigné un cèdre aujourd'hui.

b ? Fred a élagué un cèdre. Il a soigné un arbre aujourd'hui.

c ? Fred a élagué un arbre. Il a soigné un cèdre (aujourd'hui).

d Fred a élagué un arbre. Plus précisément, il a soigné un cèdre.

Etant donné ces exemples de DP sans DG correspondant, on pourrait penser que la condition pour observer un DP se réduise à ce que chaque élément $\mathrm{X}_{2}{ }^{r}$ de $P_{2}$ soit dans une relation lexico-sémantique avec l'élément correspondant $\mathrm{X}_{1}{ }^{r}$ de $P_{1}$ (s'il existe), quelle que soit cette relation (hyperonymie aussi bien qu'hyponymie, anaphore, identité ou synonymie). Une telle condition peut être intuitivement formulée en termes "d'unification" entre les deux DRS associées. Par exemple, dans (28c), l'unification des DRS des deux phrases est possible et débouche sur la DRS correspondant à Fred a élagué un cèdre (aujourd'hui) rassemblant les hyponymes de l'une ou l'autre phrase. Une condition basée sur l'unification suppose que l'ordre 
des phrases n'est pas pertinent si tant est que $P_{2}$ apporte au moins une information nouvelle comparée à l'information transmise dans $P_{l}$. Et de fait, il existe des exemples de DP dans lesquels l'ordre des phrases peut être inversé : (29a) et (29b), qui diffèrent uniquement par l'ordre des phrases (et des relations anaphoriques), sont tous les deux des DP tout à fait naturels avec marcher < aller. (29a) est un DP dans lequel $P_{2}$ apporte l'information que Fred a marché jusqu'à la gare et que cette marche a pris une heure. (29b) est un DP dans lequel $P_{2}$ apporte l'information que la marche de Fred l'a mené à la gare ${ }^{10}$.

(29)a Fred est allé à la gare. Il a marché pendant une heure.

b Fred a marché pendant une heure. Il est allé à la gare.

Néanmoins, une condition basée sur l'unification pour observer un DP n'est pas adéquate : (17a) ou (30a), dans lequel Pred $_{2}$ est un hyponyme de $\operatorname{Pred}_{1}$ ( élaguer < soigner), tandis qu'Arg ${ }_{2}^{\text {Patient }}$ est un hyperonyme d'Arg ${ }_{1}^{\text {Patient }}$ (arbre > cèdre), n'est définitivement pas un DP : il ne peut être compris comme mettant en jeu un unique événement (et un unique arbre). L'insertion d'un marqueur de coréférentialité n'aide pas : (30b) avec plus précisément est incohérent. Or, dans (30a), l'unification des DRS des deux phrases est possible et débouche sur la DRS associée à Fred a élagué un cèdre (aujourd'hui), comme c'est le cas pour (28c). Le contraste entre (28c) et (30a) demande à être expliqué.

(30)a \#Fred a soigné un cèdre. Il a élagué un arbre (aujourd'hui).

b \#Fred a soigné un cèdre. Plus précisément, il a élagué un arbre.

Pour conclure, $C_{P D}$ est limité aux DP avec un DG correspondant. Il existe d'autres DP sans DG correspondant pour lesquels $C_{P D}$ n'est pas respecté. Dans ces DP, $P_{2}$ peut inclure un hyperonyme d'un élément correspondant de $P_{1}$. Cependant, la présence d'hyperonymes dans $P_{2}$ est contrainte. Par conséquent, une condition basée sur l'unification est trop faible. Il doit au moins être postulé qu'un argument indéfini dans $P_{2}$ ne peut pas être un hyperonyme de l'argument correspondant dans $P_{1}$ quand Pred $_{2}$ est un hyponyme de Pred (afin d'exclure (30)). En tout cas, il est clair que les conditions pour observer un DP sont basées sur des connaissances linguistiques. Nous notons $C_{\text {part }}$ cet ensemble de conditions.

Avant de conclure cette section sur les DP, une remarque sur la notion "de nouvelle information" à propos des GN démonstratifs.

\subsection{GN démonstratifs dans les DP}

Dans tous les exemples que nous avons présentés jusqu'ici, $\operatorname{Arg}_{2}{ }^{\text {Patient }}$ (si présent) était une anaphore pronominale d'Arg ${ }_{1}^{\text {Patient }}$ ou un GN indéfini (comme dans (31a)). Nous allons maintenant examiner les cas où $\mathrm{Arg}_{2}$ Patient est un GN défini, (31b), ou un GN démonstratif, (31c). (31b) est un DP acceptable seulement dans un contexte où il existe un unique cèdre. (31c) est un DP acceptable seulement dans une utilisation déictique du GN. Dans une utilisation non déictique, (31c) n'est pas un DP et est incohérent. Dorénavant, l'utilisation déictique des GN démonstratifs est mise de côté, seule l'utilisation anaphorique est considérée.

(31)a Fred a élagué un arbre. Il a élagué un cèdre.

b Fred a élagué un arbre. Il a élagué le cèdre.

c \# Fred a élagué un arbre. Il a élagué ce cèdre.

\footnotetext{
${ }^{10}$ Dans (29), l'événement de marche et l'événement "d'aller" sont compris comme coréférents bien que le premier soit atélique tandis que le second est télique. L'unification des deux phrases en une seule phrase donne Fred a marché à la gare en une heure qui est télique avec l'adjoint en une heure (et non pendant une heure).
} 
Les discours (32), où $\operatorname{Arg}_{2}{ }^{\text {Patient }}$ est un GN démonstratif, sont tous des DP acceptables dans lesquels la nouvelle information dans $P_{2}$ vient de hier.

(32)a Fred a élagué un cèdre. Il a élagué cet arbre hier.

b Fred a élagué un arbre. Il a élagué cet arbre, qui est très vieux, hier.

c Fred a élagué un arbre. Il a élagué ce cèdre hier.

La coréférence entre les deux patients est de type généralisation dans (32a) puisque arbre est un hyperonyme de cèdre (cet arbre $=\mathrm{GEN}($ un cèdre)). Par contre, elle est de type particularisation dans (32b) et (32c). Dans (32b), la relative explicative apporte une information nouvelle (cet arbre, qui est très vieux, $=$ PART(un cèdre)). Dans (32c), le nom principal d'Arg ${ }_{2}^{\text {Patient }}$ étant un hyponyme du nom principal de $\operatorname{Arg}_{1}{ }^{\text {Patient }}$ apporte une information nouvelle (ce cèdre = PART( $u n$ arbre $))^{11}$. Mais quel que soit le type de coréférence entre les deux patients (généralisation ou particularisation), la présence de hier est obligatoire dans tous les exemples de (32) (voir le contraste entre (32c) et (31c)). Cela revient à dire que même quand un GN démonstratif anaphorique apporte une information nouvelle sur l'entité à laquelle il réfère, il n'apporte pas d'information nouvelle sur l'événement en jeu. Cette situation contraste avec celle observée pour les GN indéfinis : dans (31a), un cèdre apporte une information nouvelle sur l'arbre concerné et cette information est considérée comme nouvelle par rapport à l'événement d'élagage. De même pour les GN définis, (31b).

En résumé, une distinction doit être faite entre information nouvelle sur une entité $x$ en soi et information nouvelle sur une entité $x$ en tant que participant à une éventualité.

\section{Relations de discours}

Il est probable que tout chercheur travaillant sur les relations de discours poserait que la relation de discours entre les deux phrases d'un DP est Élaboration. Cependant, nous allons montrer qu'il est nécessaire de postuler l'existence d'une nouvelle relation de discours, appelée "Particularisation", qui implique une relation de coréférence événementielle. Auparavant, une remarque terminologique : "particularisation" (abrégé en PART) désigne un type de coréférence défini dans la Section 1 ; "Particularisation" (avec une majuscule) désigne une relation de discours entre deux phrases. Ces deux notions sont liées en SDRT [Asher, 1993] de la façon suivante où $\pi_{\mathrm{i}}$ est l'étiquette pour la DRS $k_{i}$ représentant la phrase $P_{i}$ :

$$
k_{2}=\operatorname{PART}\left(k_{1}\right) \Leftrightarrow \text { Particularisation }\left(\pi_{1}, \pi_{2}\right)
$$

La relation de discours Élaboration est définie de façons diverses [Hobbs, 1979, Mann et Thompson, 1988, Asher et Lascarides, 1995], mais quelle que soit sa définition, cette relation de discours n'implique pas de coréférence événementielle. Illustrons ce point avec un exemple "classique" d'Élaboration, (33a) ou sa version plus courte, (33b). La première phrase dénote un événement $e_{1}$ qui est "élaboré" dans la (les) phrase(s) suivantes qui apporte(nt) une information nouvelle sur une ou plusieurs phases (sous-événements) de $e_{1}$. Aucune coréférence événementielle n'est en jeu : un voyage en avion ne se réduit pas à un décollage et un atterrissage et encore moins à un simple décollage. Il existe beaucoup d'autres phases du voyage qui ne sont décrites ni dans (33a) ni dans (33b).

Nicholas est allé en avion d'Austin à Paris. Il a décollé à $6 \mathrm{~h}$. Il a atterri à $14 \mathrm{~h}$.

\footnotetext{
${ }^{11}$ (32c) exige une accomodation [Van Der Sandt, 1992] : quand $P_{2}$ est énoncé, l'interlocuteur apprend soudain que l'arbre impliqué dans $P_{1}$ est en fait un cèdre. Cet exemple n'est pas très heureux et doit être évité dans un système de génération de texte [Roussarie, 2000]. Cependant, nous le considérerons comme acceptable ici.
} 
b Nicholas est allé en avion d'Austin à Paris. Il a décollé à $6 h$.

Il est toutefois nécessaire de postuler l'existence d'une relation de discours (Particularisation) qui implique une relation de coréférence événementielle. Dans le cadre de la SDRT, la conséquence indéfaisable de Particularisation se traduit de la façon suivante :

$$
\text { Particularis ation } \quad\left(\pi_{1}, \pi_{2}\right) \rightarrow \text { Main - event }\left(\pi_{1}\right)=\text { Main - event }\left(\pi_{2}\right)
$$

En effet, seule une telle relation de discours permet de préserver les connaissances bien établies sur les référents de discours. L'argumentation est la suivante : les exemples comme (1) ou (4) sont des contre-exemples productifs au principe de la lecture existentielle d'un GN indéfini (qui entraîne que deux GN indéfinis ne sont pas coréférents). D'où deux solutions vis-à-vis de ce principe :

- $\mathrm{Au}$ vu de ces contre-exemples productifs, ce principe est abandonné et une lecture anaphorique des GN indéfinis est mise en place pour permettre la coréférence de deux GN indéfinis. Cette solution ne semble pas appropriée puisque ce principe est toujours vrai sauf dans les DP (et les DG et les quelques exceptions bien connues mentionnées dans la note 5).

- Ce principe n'est pas abandonné. Les deux GN indéfinis de (1) reçoivent une lecture existentielle : deux référents de discours $x$ et $y$ avec des conditions complètes $\operatorname{arbre}(x)$ et cèdre $(y)$ sont introduits. La relation de coréférence $x=y$ est établie grâce à la relation de discours Particularisation. Cette relation de discours implique une relation de coréférence événementielle qui implique à son tour que les arguments avec le même rôle thématique sont coréférents. Autrement dit, c'est parce que Particularisation est établie entre les deux phrases de (1) que l'on sait que les deux phrases réfèrent au même événement et que donc les deux patients un arbre et un cèdre sont coréférents, c'est-à-dire $x=y$.

Cette seconde solution semble nettement plus adéquate. Elle soulève immédiatement la question suivante : comment établir que la relation de discours dans (1) est Particularisation (ce qui revient à établir que (1) est un DP) ? La réponse à cette question repose sur des connaissances linguistiques : les deux phrases d'un DP respectent des contraintes linguistiques fortes qui ont été décrites dans la Section 5. Cet ensemble de contraintes, noté $C_{\text {part }}$, permet de calculer que la relation de discours est Particularisation. En SDRT, la règle est la suivante (où $>$ est le symbole pour l'implication par défaut) :

$$
\left(\tau, \pi_{1}, \pi_{2}\right) \wedge C_{\text {part }}>\text { Pa r tic u la r isation }\left(\pi_{1}, \pi_{2}\right)
$$

En résumé, il est nécessaire de postuler l'existence d'une nouvelle relation de discours, Particularisation, qui implique une relation de coréférence événementielle entre l'événement $e_{\boldsymbol{I}}$ décrit dans la première phrase et l'événement $e_{2}$ décrit dans la seconde. Cette relation de discours diffère d'Élaboration pour laquelle seule(s) une ou quelques phases (sous-événements) de $e_{1}$ sont spécifiées. Néanmoins, on peut voir Particularisation comme un cas particulier d'Élaboration. Dans le même ordre d'idées, il est nécessaire pour les DG de postuler l'existence d'une nouvelle relation de discours, Généralisation, qui implique une relation de coréférence événementielle et que l'on peut voir comme un cas particulier de Reformulation.

\section{Conclusion}

À la différence de la coréférence entre objets, la coréférence événementielle est une question qui n'a pas été vraiment examinée (à part pour les groupes (pro)nominaux anaphorisant une phrase). Cependant, la coréférence événementielle est une notion centrale pour plusieurs types de 
discours. Cet article porte sur les discours généralisants et particularisants. Dans [Danlos, 2000, Danlos et Gaiffe, 2000], nous avons montré qu'une relation de coréférence événementielle de type particularisation est en jeu dans des discours causaux analysés avec la relation de discours "Explication", tandis que la généralisation est en jeu dans des discours causaux analysés avec "Résultat". La coréférence événementielle est donc une question de cohésion (la coréférence, [Halliday et Hasan, 1976]), qui s'avère être cruciale pour une question de cohérence (établissement de relations de discours).

Nous avons montré que la coréférence événementielle dans les DP exige une nouvelle relation de discours, Particularisation, plus spécifique qu'Élaboration qui est généralement employée pour analyser des discours comme (1). Particularisation (et sa relation duale Généralisation) est établie sur la base de contraintes linguistiques fortes (contraintes lexicosémantiques entre éléments avec le même rôle). Elle permet d'établir une relation de coréférence événementielle bien que les événements dénotés par les phrases soient quantifiés existentiellement. Cette relation de coréférence événementielle implique la coréférence de participants ayant le même rôle thématique, même s'ils sont quantifiés existentiellement.

\section{Bibliographie}

Asher, N. (1993). Reference to Abstract Objects in Discourse. Kluwer, Dordrecht.

Asher, N. and Lascarides, A. (1995). Lexical disambiguation in a discourse context. Journal of Semantics, 12(12):69-108.

Corblin, F. (1994). La condition de nouveauté comme défaut. Faits de langues (Presses Universitaires de France), (4):147-160.

Corblin, F. (1995). Les formes de reprise dans le discours, Anaphores et chaînes de référence. Presses Universitaires de Rennes, Rennes.

Danlos, L. (2000). Event coreference in causal discourses. In Bouillon, P. and Busa, F., editors, Meaning of Word. Cambridge University Press.

Danlos, L. and Gaiffe, B. (2000). Coréférence événementielle et relations de discours. In Actes de la conférence Traitement automatique des Langues Naturelles (TALN'00), Lausanne.

Halliday, M. A. K. and Hasan, R. (1976). Cohesion in English. Longman, London.

Hobbs, J. (1979). Coherence and coreference. Cognitive Science, (3):67-90.

Humphreys, K., Gaizauskas, R., and Azzam, S. (1997). Event coreference for information extraction. In Proceedings of the Workshop on Operational Factors in Pratical, Robust Anaphora Resolution for Unrestricted Texts, 35th Meeting of ACL, Madrid.

Kamp, H. and Reyle, U. (1993). From Discourse to Logic. Introduction to Modeltheoretic Semantics of Natural Language, Formal Logic and Discourse Representation Theory. Kluwer Academic Publishers, Dordrecht, The Netherlands.

Mann, W. C. and Thompson, S. A. (1988). Rhetorical structure theory: Toward a functional theory of text organization. Text: An Interdisciplinary Journal for the Study of Text, 8(2).

Moens, M. and Steedman, M. (1988). Temporal ontology and temporal reference. Computational Linguistics, 14:15-28. 
Pustejovsky, J. (1995). The generative Lexicon. The MIT Press.

Rossari, C. and Jayez, J. (1996). Donc et les consécutifs. Des systèmes de contraintes différentiels. Lingvistica Investigationes, XX(1):117-143.

Roussarie, L. (2000). Un modèle théorique d'infèrences de structures sémantiques dans le cadre de la génération automatique de textes.Thèse de l'Université Denis Diderot, Paris 7.

Van Der Sandt, R. (1992). Projection as anaphora resolution. Journal of Semantics, 9:333-377.

Webber, B. (1988). Discourse deixis: Reference to discourse segments. In Proceedings of $A C L ' 1988$, pages 113-123, Buffalo, NY. 